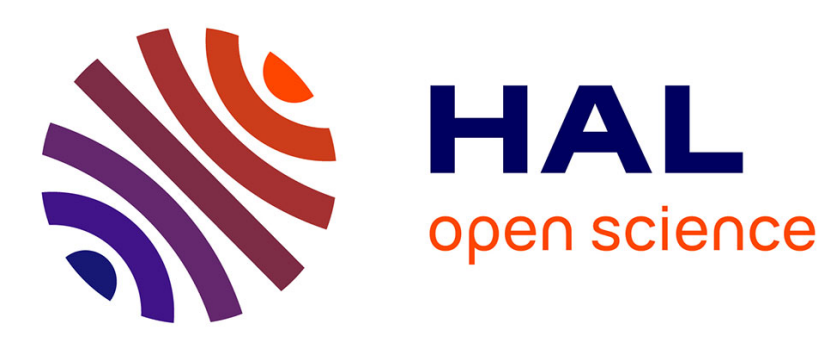

\title{
DFTB-Assisted Global Structure Optimization of 13- and 55-Atom Late Transition Metal Clusters
}

\author{
Maxime van den Bossche
}

\section{To cite this version:}

Maxime van den Bossche. DFTB-Assisted Global Structure Optimization of 13- and 55-Atom Late Transition Metal Clusters. Journal of Physical Chemistry A, 2019, 123 (13), pp.3038-3045. 10.1021/acs.jpca.9b00927 . hal-02181710

\section{HAL Id: hal-02181710 https://hal.sorbonne-universite.fr/hal-02181710}

Submitted on 12 Jul 2019

HAL is a multi-disciplinary open access archive for the deposit and dissemination of scientific research documents, whether they are published or not. The documents may come from teaching and research institutions in France or abroad, or from public or private research centers.
L'archive ouverte pluridisciplinaire HAL, est destinée au dépôt et à la diffusion de documents scientifiques de niveau recherche, publiés ou non, émanant des établissements d'enseignement et de recherche français ou étrangers, des laboratoires publics ou privés. 


\title{
DFTB-Assisted Global Structure Optimization of 13- and 55-Atom Late Transition Metal Clusters
}

\author{
Maxime Van den Bossche*(i) \\ CNRS, Institut des NanoSciences de Paris, UMR 7588, Paris F-75005, France \\ Supporting Information
}

ABSTRACT: Finding globally optimal structures of nanoclusters is critically important to understand their physicochemical properties but remains prohibitively expensive even with comparatively efficient density functionals. Semiempirical methods such as density functional tight-binding (DFTB), on the other hand, offer a better accuracy-efficiency trade-off but require suitable parametrization. In the present work, we present a largely automatic scheme where, starting from initial guesses based on bulk properties, the atomic confinement, and repulsive potentials are further refined so as to accurately represent the potential energy landscapes of 13and 55-atom nanoclusters of the late transition metals $(\mathrm{Ni}$,

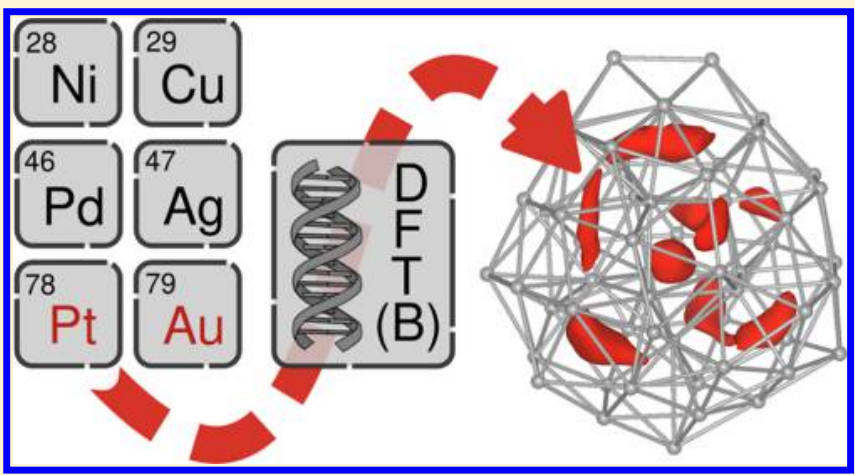
$\mathrm{Cu}, \mathrm{Pd}, \mathrm{Ag}, \mathrm{Pt}$, and $\mathrm{Au}$ ). With the exception of $\mathrm{Ni}_{13}, \mathrm{Ni}_{55}$, $\mathrm{Cu}_{55}$, and $\mathrm{Ag}_{55}$, low-symmetry (often disordered) structures are found to be preferred over the symmetric icosahedral arrangement. Similar to what has been previously reported for $\mathrm{Au}_{55}$, the lowest-energy $\mathrm{Pt}_{55}$ structures also appear to contain small cavities below the outer shell.

\section{INTRODUCTION}

Metal clusters have found numerous applications in a variety of technologies, including heterogeneous catalysis as well as medicinal and optoelectronic devices. ${ }^{1}$ Their usefulness stems from a high specific surface area, together with chemical and physical properties that are different from those of extended metal surfaces and that often vary with the size and shape of the cluster. ${ }^{2}$ Nanometer-sized Au particles on oxide supports, for example, can turn the otherwise inert $\mathrm{Au}$ into a potent oxidation catalyst. ${ }^{3}$

Insights into the most probable structural arrangements of such clusters are then of prime importance for a detailed understanding of their properties and for the rational design of further improved materials. ${ }^{4}$ While experimental methods can probe many characteristics of both free and supported metal clusters, complete structure determination is very difficult to achieve.

Atomistic simulations, on the other hand, offer the desired resolution but require a thorough search of the potential energy landscape to locate the most relevant (e.g., lowest energy) structures. ${ }^{5}$ Though such searches would preferentially be carried out using accurate and transferable electronic structure methods such as density functional theory (DFT), the computational cost and time-to-solution rapidly become intractable as the number of metal atoms is increased. While global optimization (GO) of clusters with a few tens of atoms can nowadays be performed directly at the DFT level ${ }^{6,7}$ given ample resources, larger cluster sizes are likely to remain out of reach for a considerable time to come. The inclusion of a support material further reduces the currently accessible cluster size to about 10 metal atoms. ${ }^{8-10}$

Along with continued development of more efficient GO algorithms, ${ }^{11-13}$ there is therefore a pressing need for alternative descriptions of the potential energy landscape which trade an acceptable decrease in accuracy for a considerable increase in speed. Interatomic potentials such as embedded atom methods (EAM) and Gupta potentials, for example, present a very low computational cost. The same is generally true of machine learning approaches, such as neural networks, ${ }^{14,15}$ though the large amount of required training data evidently impacts the total cost.

Approximate electronic structure methods, such as density functional tight-binding (DFTB), constitute a third option with an appealing set of advantages. The cost per force call is 2-3 orders of magnitude below that of efficient GGA-DFT implementations, ${ }^{16}$ while retaining an explicit (though strongly simplified) description of the electronic structure. Furthermore, generating suitable DFTB parametrizations is comparatively straightforward and lends itself well to automatization. $^{17,18}$ Lastly, DFTB can be made to reproduce DFT properties more accurately than common interatomic potentials, which will also be illustrated here.

Even though one may have expected metallic compounds to present significant challenges to the two-center and minimal- 
basis approximations in standard DFTB, encouraging results have been previously reported for many transition metals, such as $\mathrm{Fe},{ }^{19-22} \mathrm{Au}^{23-26} \mathrm{Ag}^{26,27} \mathrm{Mo}^{28}$ and $\mathrm{Pt}-\mathrm{Ru}$ alloys ${ }^{29}$ and other small transition metal clusters. ${ }^{30}$

Compared to these previous studies, the present work describes a DFTB parametrization scheme that is more specifically tailored toward and integrated into the GO problem at hand. After a brief outline of DFTB in section 2, this approach is described in detail in section 3. The results are then presented and discussed for the 13- and 55-atom clusters of the late transition metals $(\mathrm{Ni}, \mathrm{Cu}, \mathrm{Pd}, \mathrm{Ag}, \mathrm{Pt}, \mathrm{Au})$ in section 5 , followed by concluding remarks in section 6 . The two cluster sizes have been chosen so as to evaluate the prevalence of the icosahedral motif relative to more disordered arrangements.

\section{DFTB BACKGROUND}

DFTB constitutes an approximation to LCAO-DFT in which the computational cost is reduced through (i) the use of a small (preferentially minimal) set of optimized basis functions $\phi$, (ii) the approximate evaluation of Hamiltonian matrix elements, and (iii) the introduction of an empirical "repulsive" potential to represent the remaining contributions to the DFT total energy. In self-consistent charge (SCC) DFTB, the expression for the total energy is then given by ${ }^{31}$

$$
E_{\text {tot }}=E_{\text {band }}+E_{\text {scc }}+E_{\text {rep }}
$$

The band structure energy $E_{\text {band }}$ is calculated by summing the eigenvalues $\epsilon_{i}$ of the occupied states gathered from a selfconsistent solution to the secular equations

$$
\left[\mathcal{H}^{0}+\Delta \mathcal{H}-\epsilon \mathcal{S}\right] C=0
$$

where $\mathcal{H}^{0}$ is the Hamiltonian matrix consistent with a superposition of atomic densities $\rho^{0}, \Delta \mathcal{H}$ its electrostatic corrections due to charge density fluctuations, and with the overlap and coefficient matrices $\mathcal{S}$ and $C$.

The $\mathcal{H}_{\mu \nu}^{0}=\left\langle\phi_{\mu}\left|-\frac{1}{2} \nabla^{2}+V_{\text {eff }}\left[\rho^{0}\right]\right| \phi_{\nu}\right\rangle$ matrix elements are approximated by only considering the contributions to the effective potential $V_{\text {eff }}$ of the atomic centers $a$ and $b$ on which the orbitals $\mu$ and $\nu$ are located. In the density superposition scheme $V_{\text {eff }}\left[\rho^{0}\right]$ then reduces to $V_{\text {eff }}\left[\rho_{a}^{0}+\delta_{a b} \rho_{b}^{0}\right]$. As a result, the one-center integrals correspond to $\mathcal{H}_{\mu \nu}^{0}=\delta_{\mu \nu} \epsilon_{\mu}$ (with $\epsilon_{\mu}$ the corresponding eigenvalue in the isolated atom) and $\mathcal{S}_{\mu \nu}=\delta_{\mu \nu}$. The two-center $\mathcal{H}^{0}$ and $\mathcal{S}$ integrals are computed beforehand and tabulated as a function of distance and orientation, making use of the Slater-Koster transformation rules. ${ }^{32}$ The required basis functions $\phi$ and atomic densities $\rho^{0}$ are obtained from atomic DFT calculations with added confinement through the use of, e.g., polynomial potentials

$$
V_{\text {conf }}=\left(\frac{r}{r_{0}}\right)^{s}
$$

with empirical parameters $r_{0}$ and $s$, which can be chosen differently for each of the different orbital angular momenta and the density $\rho^{0}$.

The SCC corrections $\Delta \mathcal{H}$ and $E_{\text {scc }}$ to the Hamiltonian and the total energy (described in detail in refs 31 and 33) can then be constructed after approximating the charge density fluctuations as atom-centered, exponentially decaying charge distributions. The $l$-dependent atomic charges are convention- ally deduced from a Mulliken population analysis of the occupied electronic states. The decay lengths are determined from the Hubbard parameters $U_{l}$, which can in turn be calculated as the difference between the electron removal and electron addition energies from atomic DFT calculations.

The final $E_{\text {rep }}$ term is intended to contain all remaining contributions to the total energy (such as core-core repulsion) and, to a certain degree, correct the approximations made in the $E_{\mathrm{band}}$ and $E_{\mathrm{scc}}$ terms. A major convenience of this decomposition is that a set of smooth, short-ranged potentials for each element pair generally succeeds well in representing $E_{\text {rep, }}{ }^{31,34}$ i.e.,

$$
E_{\text {rep }}=\sum_{i<j} V_{\text {rep }, i j}\left(r_{i j}\right)
$$

Fitting $V_{\text {rep }}$ becomes a straightforward linear regression problem when using polynomial functions, e.g.

$$
V_{\text {rep }}(r)=\sum_{p=2}^{6} c_{p}\left(r_{\text {cut }}-r\right)^{p}
$$

The cutoff radius $r_{\text {cut }}$ is typically chosen in between the firstand second-nearest neighbor distances of the element pair in question.

\section{METHODS}

In seeking to accelerate GO searches using DFTB, one faces the problem that the DFTB parametrization needs to be most accurate for the low-lying minima of the DFT potential energy surface (PES). Including information on low-energy structures in the DFTB training set, however, seems to itself require the solution of the GO problem at hand. The previously published tight-binding approximation enhanced global optimization (TANGO) approach $^{35}$ addresses this bootstrapping problem by creating an initial, preliminary parametrization (using, e.g., a randomly generated structure database), which is then iteratively refined based on DFT single-point calculations of low-energy structures suggested by the same parametrization.

The same approach will be used here, though with certain simplifications (compared to the TANGO searches for bulk crystal structures in ref 35 ), made possible by previous studies on DFTB parametrizations for metal clusters. Most notably, Fihey and co-workers ${ }^{25}$ have described a parametrization procedure for $\mathrm{Au}$ clusters which performs quite adequately given its relative simplicity. This process is depicted in the top panel of Figure 1 and will be adopted here to generate the initial DFTB parametrization in the TANGO runs.

First, the eigenvalues $\epsilon_{l}$ and Hubbard parameters $U_{l}$ of the isolated, spherical, and spin-unpolarized atom are obtained from all-electron atomic DFT calculations, here performed using the ATOM code in the CP2K suite ${ }^{36-38}$ using the Douglas-Kroll-Hess method ${ }^{39-41}$ to include scalar-relativistic effects.

The parameters describing the confinement of the s, p, and $d$ orbitals and the electron density $\rho^{0}$ are then optimized using the gradient-less COBYLA algorithm ${ }^{42}$ so that the DFTB band structures of the bulk FCC, BCC and SC crystals match the corresponding DFT band structures. Following ref 25, most importance is attached to the band structure around the Fermi level, which is done here by assigning Boltzmann-distributed weights 


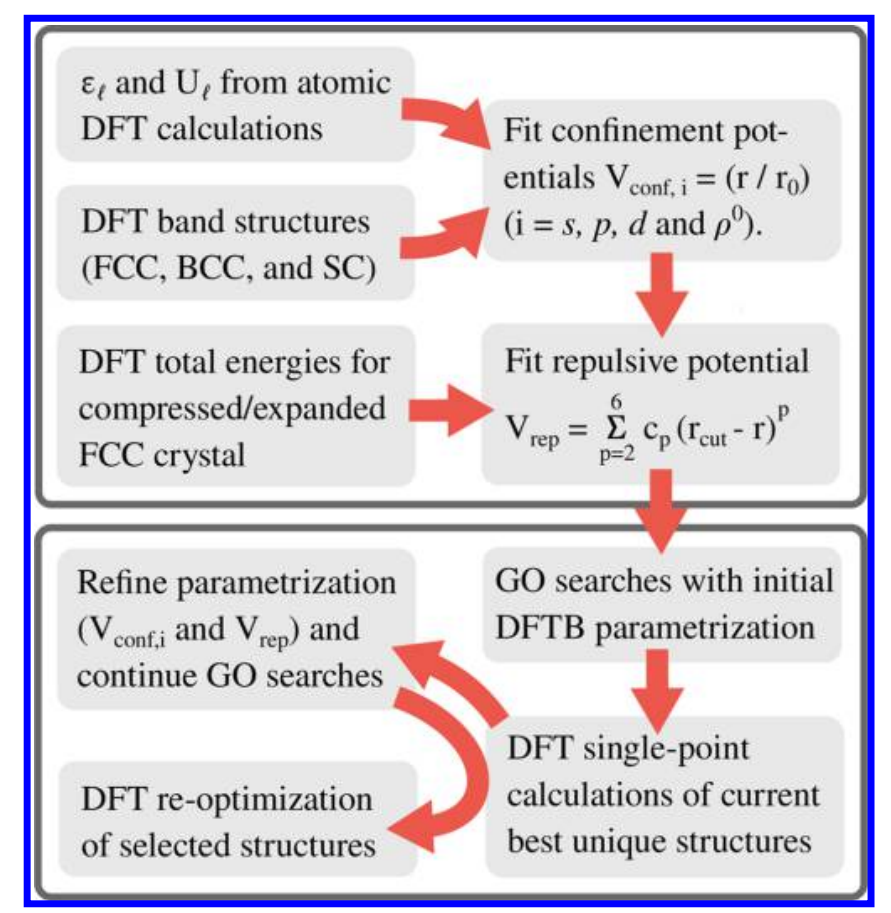

Figure 1. Overview of the DFTB parametrization plus global optimization strategy for the studied transition metal clusters.

$$
w=\exp \left(-\frac{\left|\epsilon-E_{\text {Fermi }}\right|}{k_{\mathrm{B}} T}\right)
$$

with $k_{\mathrm{B}} T$ set equal to $1.5 \mathrm{eV}$. The band structure fitting and associated atomic DFT calculations under confinement and Slater-Koster tabulation are performed using the Hotcent $\operatorname{code}^{79}$ (commit ab939fe2) developed as part of this work.

To complete the initial DFTB parametrization, the repulsive potential is calculated based on the difference between the DFT total energy and the repulsionless DFTB total energy of the FCC structure for interatomic distances ranging from 1.4 to 3 times the covalent radius of the metal.

The next phase, represented by the bottom panel in Figure 1 , starts with a set of comparatively short GO searches to identify the type of geometries favored by the present DFTB parametrization. The 100 best unique structures are then subjected to DFT single-point calculations, thereby creating a training set for further refinement of the DFTB model. Both the confinement and repulsive potentials are optimized to lower the total residual. For reasons of numerical stability, however, the confinement potentials are here restricted to the harmonic kind $(s=2)$, while allowing $r_{0}$ to vary. The total residual is given by

$$
\begin{aligned}
R_{\mathrm{SSQ}}= & \sum_{i=1}^{M} \omega_{i}\left[\left(E_{\mathrm{coh}, i}^{\mathrm{DFT}}-E_{\mathrm{coh}, i}^{\mathrm{DFTB}}-C\right)^{2}\right. \\
& \left.+\omega_{f} \sum_{j=1}^{N}\left\|\bar{F}_{i, j}^{\mathrm{DFT}}-\bar{F}_{i, j}^{\mathrm{DFTB}}\right\|^{2}\right]
\end{aligned}
$$

with $E_{\mathrm{coh}}, i$ the cohesive energy of structure $i$ and $\bar{F}_{i, j}$ the force on atom $j$ of structure $i$. The weights $\omega_{i}$ are distributed according to the relative DFT total energies, following a Boltzmann distribution as in eq 6 with $k_{\mathrm{B}} T$ set to $0.1 \mathrm{eV}$ times the number of atoms. An additional scaling factor $\omega_{f}$ of $0.1 \AA$ / atom is applied to the force residuals, which is found to provide a suitable balance between the description of energetical and geometrical properties. Note that the procedure allows for a constant shift $C$ of the DFTB cohesive energies, so as to prevent accuracy losses due to consistent under- or overbinding tendencies which do not affect the energetical ordering for a given cluster size. The smoothness of the $V_{\text {rep }}$ curve is assured by weak $\mathcal{L}_{2}$ regularization of the linear regression problem with a regularization factor of $10^{-10}$. The repulsive potential fitting is performed using the TANGO $\operatorname{code}^{80}$ (commit b1 c5bcb) with a polynomial form of $V_{\text {rep }}(r)$ as shown in eq 5 .

Lastly, the GO searches are resumed with the refined DFTB parametrization and pursued more extensively so as to retrieve the lowest-lying minima on the DFTB PES. Again, the 100 best unique structures are selected and subjected to DFT single-point calculations. On the basis of the obtained DFT total energies, the 25 lowest-energy structures are reoptimized at the DFT level until the residual forces are less than $0.05 \mathrm{eV} / \AA$.

\section{COMPUTATIONAL DETAILS}

4.1. DFT Calculations. All metal cluster DFT calculations are executed using the GPAW ${ }^{43,44}$ code using a plane-wave basis set with a kinetic energy cutoff of $600 \mathrm{eV}$. The Brillouinzone integration is restricted to the $\Gamma$ point and the SCF converge is facilitated by Fermi-Dirac smearing with a width of $20 \mathrm{meV}$. The PBEsol functional ${ }^{45}$ is chosen for the description of electronic exchange and correlation, as this functional has been found to be more accurate than other gradient-corrected functionals for small $\mathrm{Au}$ clusters, ${ }^{46}$ in addition to generally providing accurate lattice parameters as well as jellium surface energies. ${ }^{45,47,48}$ Note that all atomic DFT calculations as well as the density superposition calculations of the Hamiltonian integrals are also performed using the PBEsol functional and with addition of scalarrelativistic corrections. The number of explicitly treated valence electrons is limited to $10(\mathrm{Ni}, \mathrm{Pd}, \mathrm{Pt})$ and $11(\mathrm{Cu}$, $\mathrm{Ag}, \mathrm{Au})$ per atom using the standard projector augmentedwave $^{49}$ setups in GPAW regenerated for the PBEsol functional. It should be noted that spin polarization has not been included in the $\operatorname{DFT}(\mathrm{B})$ calculations unless stated otherwise.

4.2. DFTB Calculations. All DFTB calculations are carried out using the DFTB $+\operatorname{code}^{50}$ (version 17.1) with a maximum angular momentum $l_{\max }=2$ and angular momentum dependent Hubbard values. The applied Fermi-Dirac smearing width amounts to $500 \mathrm{~K}(42 \mathrm{meV})$.

Though timings usually vary across different hardware and software environments, the relative speed of DFTB can be appreciated by looking at the CPU time per force call. For the 55-atom clusters, we obtain approximately one second per DFTB force call on a single CPU, compared to ca. $300 \mathrm{~s}$ per force call using 32 CPUs with the above DFT setup.

4.3. Global Optimization. The GO searches are performed using the genetic algorithm (GA) framework ${ }^{10,51}$ in the atomic simulation environment (ASE). ${ }^{52,53}$ Each GA run involves the following steps:

1. Randomly generating 20 clusters with coordinates within a sphere of $110 \AA^{3}$ (13 atoms) and $460 \AA^{3}$ (55 atoms), corresponding to a number density of about 0.12 atoms per $\AA^{3}$. The clusters are placed in cubic unit cells with 


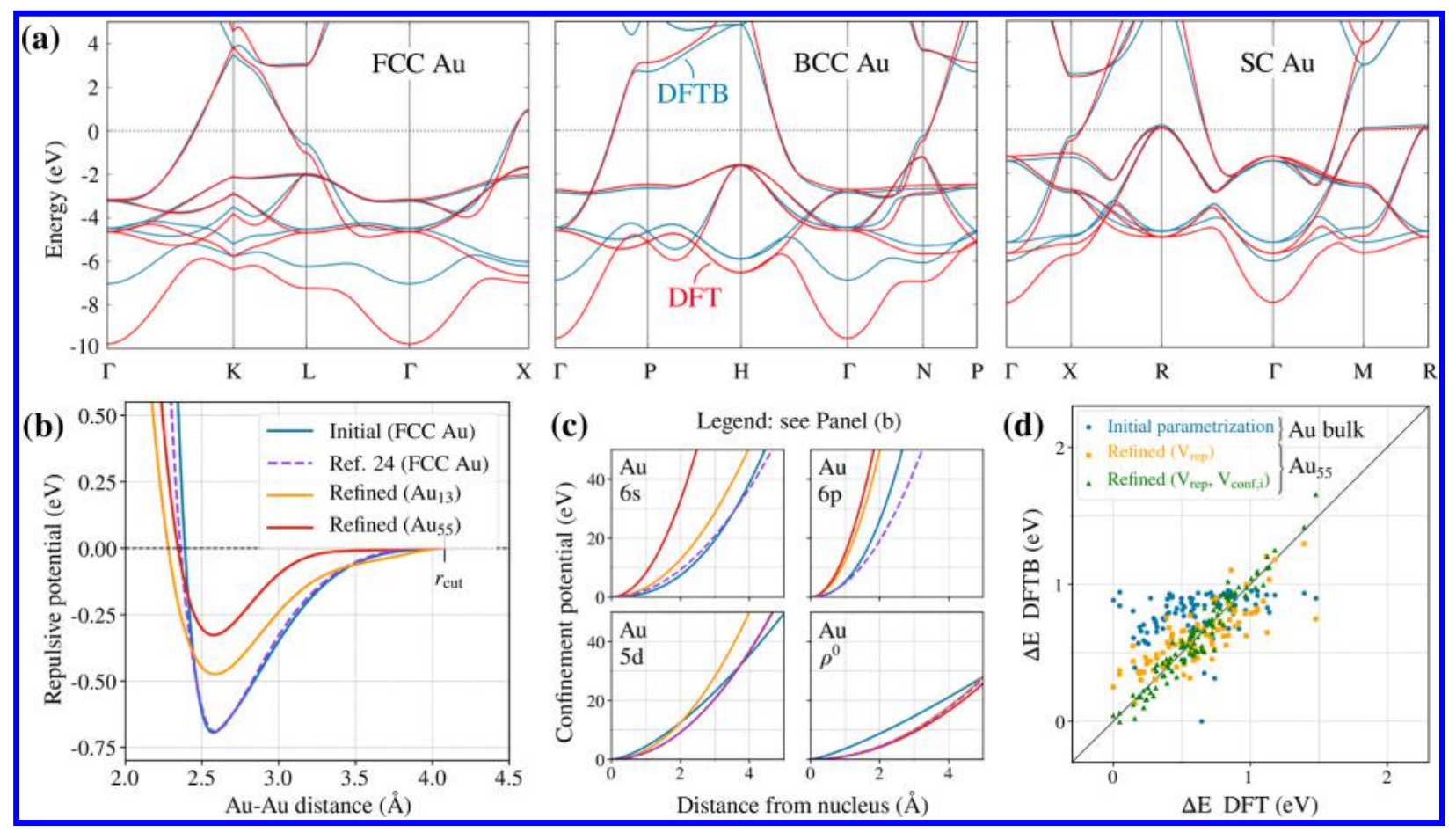

Figure 2. Panel a: Calculated band structures for FCC, BCC and SC Au using DFT (red) and DFTB with optimized confinement potentials (blue). The eigenvalues are referenced with respect to the Fermi energy. Panel b: Repulsive potential between Au atoms in the initial parametrization based on bulk $\mathrm{Au}$ properties (blue) and in the refined parametrizations for $\mathrm{Au}_{13}$ (orange) and $\mathrm{Au}_{55}$ (red) where the repulsive and confinement potentials have been reoptimized. The curve obtained by Fihey and co-workers ${ }^{25}$ is drawn with purple dashes. Panel c: Analogous representation of the confinement potentials for the different valence states of $\mathrm{Au}$ and its reference atomic density. Panel d: Parity diagrams comparing the relative DFTB and DFT energies for the 100 best unique $\mathrm{Au}_{55}$ cluster structures obtained from the first series of GO searches using the initial parametrization based on bulk Au properties (blue dots). The DFTB results obtained after refitting $V_{\text {rep }}$ only (orange) and both $V_{\text {rep }}$ and $V_{\text {conf, i }}($ green) are also shown.

cell lengths of 16 and $25 \AA$ for 13- and 55-atom clusters, respectively.

2. Subjecting the initial structures to local minimization with DFTB using the BFGS algorithm ${ }^{54}$ until the largest forces are less than $0.03 \mathrm{eV} / \AA$. The set of relaxed structures constitutes the initial population.

3. At each GA iteration, a new candidate is created by the action of the genetic operators on one or two members of the population (randomly selected via the same procedure as in ref 51), followed by the same local minimization procedure as above. The genetic operator is chosen among cut-and-splice pairing, rattle mutation and mirror mutation with $85 \%, 10 \%$, and $5 \%$ probability, respectively. The population is then updated so as to always contain the 20 current best unique structures. Structural uniqueness is evaluated using fingerprint functions. $^{12}$

For the 13-atom clusters, the number of GA iterations is set to 100 for the search with the initial DFTB parametrization and to 200 using the refined parameters. The corresponding numbers for the 55-atom clusters amount to 500 and 1000, respectively. To increase the probability of finding the lowestenergy minima, $16 \mathrm{GA}$ searches are run in parallel on each occasion. In the case of $\mathrm{Pt}_{55}$, the initial parametrization is found to be particularly crude (favoring very open structures with only four atoms in the inner shell), and therefore an additional $1000 \mathrm{GA}$ iterations is performed after a second reparametrization based on the 100 best unique $\mathrm{Pt}_{55}$ structures found using the first reparametrized DFTB model.

\section{RESULTS AND DISCUSSION}

First, the results specific to the DFTB parametrization procedures will be presented and discussed. Next, the obtained lowest-energy structures will be shown for the 13-atom clusters with comparison to previous studies, followed by the corresponding discussion for the 55-atom clusters.

5.1. DFTB Parametrization. Various aspects of the (re)parametrization procedure are collected in Figure 2 for the case of $\mathrm{Au}$ (corresponding results for the other elements are included in the Supporting Information).

Panel a shows the DFTB band structures (blue lines) which have been fitted to DFT (red lines) by adjustment of the confinement parameters $r_{0}$ and $s$. The reproduction is of a similar quality as in ref 25 . The obtained confinement potentials are also comparable (see the filled blue and dashed purple curves in panel c), keeping in mind that the parametrization in ref 25 has been performed on the basis of a different density functional (PBE). It should be noted, however, that the confinement parameters from ref 25 have been used here as initial guesses, and that the optimization procedure has most likely converged to the closest local minimum. The repulsive potential for Au derived from bulk FCC structures is shown as the blue curve in panel b. Again, similar to what has been obtained in ref 25 (purple dashes), the repulsive potential is in fact attractive at commonly encountered nearest neighbor distances ( $r$ between than 2 and $4 \AA$ A). Similar results are retrieved for the other elements. While attractive pair potentials are relatively uncommon and may benefit from further investigation, it should be noted that these 
need not imply a failure of, e.g., the two-center approximation (see, e.g., ref 33).

As described in section 3, the DFTB parametrizations based on the bulk metal properties are only used as initial guesses, requiring further refinement based on DFT single-point calculations of the best unique structures obtained from a first round of GO searches. The change in the repulsive and confinement potentials can be seen in panels $b$ and $c$, respectively, with results for $\mathrm{Au}_{13}$ in yellow and $\mathrm{Au}_{55}$ in red. The benefits of the reparametrization are clearly illustrated by the parity plots for $\mathrm{Au}_{55}$ shown in panel $\mathrm{d}$. The correlation between the energetical ordering in DFT and DFTB is fairly poor in the initial DFTB parametrization (blue dots), and is markedly improved after fitting the repulsive and confinement potentials to the DFT data set (green dots). Readjusting only the repulsive potential (orange dots) yields intermediate results, and it is therefore recommended to also refine the confinement parameters. Similar conclusions can be drawn from the parity diagrams of the other studied clusters, which can be consulted in section 4 of the Supporting Information.

While the combined reoptimization complicates the comparison between the 13- and 55-atom parametrizations due to possible compensation effects, the different $V_{\text {rep }}$ and $V_{\text {confi, }}$ functions for the two cluster sizes suggest that it may be difficult to find a single parameter set which yields sufficiently accurate descriptions for both sizes. Readers interested in the topic of size-transferability are referred to section 5 of the Supporting Information for additional metrics and discussion. We furthermore note that choosing a single $r_{0}$ value equal to ca. 1.85 times the covalent radius (as is common in DFTB models for main group chemistry ${ }^{55,56}$ ) is not recommended for the present materials.

5.2. $M_{13}$ Clusters. Structural models of the most stable $M_{13}$ clusters after DFT reoptimization are shown in Figure 3. Only for $\mathrm{Ni}$ the icosahedron represents the preferred arrangement, in agreement with previous calculations performed with a Gupta potential. ${ }^{58}$ Among the coinage metals, $\mathrm{Cu}$ and $\mathrm{Ag}$ prefer similar low-symmetry structures, which also bear a certain resemblance to the one found for Au. This is in agreement with previous calculations performed using the PW91 functional. ${ }^{59}$ In the case of $\mathrm{Pd}$, the same bilayer geometry with $C_{3 v}$ symmetry is retrieved as previously found using the PBE functional for a singlet multiplicity. ${ }^{60}$ The $C_{s}$ symmetrical $\mathrm{Pt}_{13}$ structure is identical to the ones proposed on the basis of PW91 ${ }^{61}$ and PBE calculations. ${ }^{61}$ It should be kept in mind, however, that the precise energetical ordering of metal clusters can be sensitive to the choice of exchange-correlation functional, as has been illustrated for $\mathrm{Au} .^{46,62-65}$

5.3. $M_{55}$ Clusters. The corresponding $M_{55}$ cluster geometries are shown in Figure 3 for $\mathrm{Pd}, \mathrm{Pt}$, and $\mathrm{Au}$, while the Mackay icosahedron is retrieved as the lowest-energy configuration for $\mathrm{Ni}, \mathrm{Cu}$, and $\mathrm{Ag}$. The latter is in agreement with experimental observations ${ }^{66-68}$ as well as a host of GO studies based on interatomic potentials. ${ }^{58,69,70}$

A different picture emerges for $\mathrm{Pd}, \mathrm{Pt}$, and $\mathrm{Au}$, which are found to prefer disordered structures. In the case of $\mathrm{Au}$, this is indeed known from photoelectron spectroscopy measurements. ${ }^{66,71}$ GO searches with a Gupta potential by Garzón and Posada-Amarillas ${ }^{72}$ have yielded a compact but indeed disordered structure, and a similar structure has been obtained by subsequent annealing runs with GGA-DFT. ${ }^{71}$ In the present work, we also find the lowest-energy $\mathrm{Au}_{55}$ cluster to be disordered, but with an otherwise quite different geometry

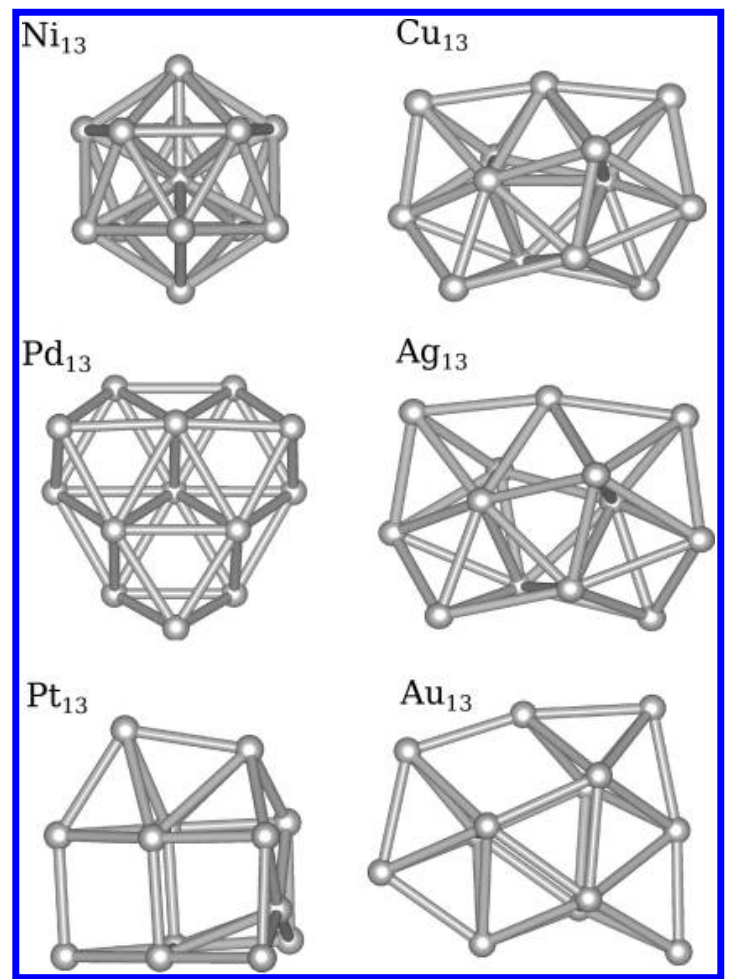

Figure 3. Lowest-energy structures obtained for the 13-atom clusters after DFT reoptimization. All structural models in this work have been rendered with VESTA. ${ }^{57}$

(shown in Figure 4). Its most prominent feature is the presence of cavities below the outer shell, which are visualized as regions of low electron density (red surfaces). The structure is in this aspect similar to the ones proposed on the basis of DFTB-GO searches by Tarrat and co-workers ${ }^{27}$ using the parametrization from ref 25 , though the voids in the present structures appear to be smaller in volume. The presence of such subsurface cavities, or "bubbles", has furthermore been previously suggested for Au clusters in the 56 to 58 atom range on the basis of DFT calculations, ${ }^{71}$ and it has been explained in terms of strong preferences for close-packed outer layers due to relativistic contraction of the 6 states. $^{71}$ Lastly, with the PBEsol functional, we calculate the $\mathrm{Au}_{55}$ cluster shown in Figure 4 to be $0.53 \mathrm{eV}$ more stable than the Garzón-PosadaAmarillas structure, $1.05 \mathrm{eV}$ more stable than the Tarrat structure, and $1.46 \mathrm{eV}$ more stable than the icosahedral arrangement. For comparison, the corresponding values using the PBE functional amount to $0.75,0.16$, and $2.03 \mathrm{eV}$, respectively. The present $\mathrm{Au}_{55}$ structure is hence also preferred

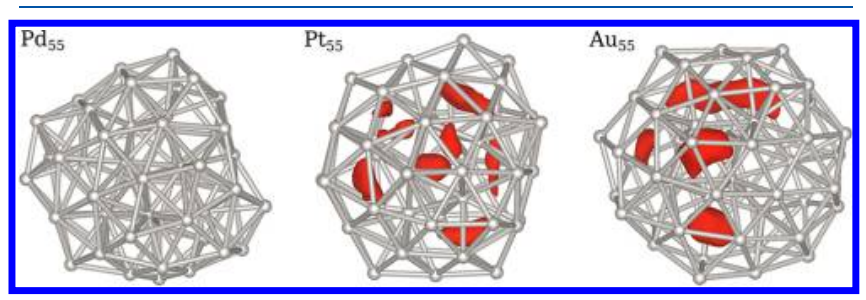

Figure 4. Lowest-energy structures obtained among the $\mathrm{Pd}_{55}, \mathrm{Pt}_{55}$, and $\mathrm{Au}_{55}$ clusters after DFT reoptimization. Cavities are highlighted by means of electron density isosurfaces (colored in red). For the other 55-atom clusters (i.e., $\mathrm{Ni}, \mathrm{Cu}$, and $\mathrm{Ag}$ ) an icosahedral ground state is retrieved. 
among the selected candidates in the PBE approximation. It is, however, quite likely that lower-energy configurations exist on the PBE energy landscape.

Platinum, the other element from the same period, is also found to prefer small (atom-sized) subsurface cavities below the outer shell (see Figure 4). This similarity is understandable as relativistic effects are almost as pronounced in $\mathrm{Pt}$ as in $\mathrm{Au}{ }^{73}$ One difference, however, is the additional presence of a small cavity in the center, with the nine atoms in the core describing a gyroelongated square pyramid. Previous calculations with interatomic potentials, by contrast, have either favored the icosahedral structure ${ }^{74}$ or lower-symmetry, but nonetheless compact structures. ${ }^{75-77}$ With the present DFT setup, the putative global minimum configuration is more stable than the icosahedron by a considerable margin $(\Delta E=-5.46 \mathrm{eV})$.

Finally, for $\mathrm{Pd}_{55}$ a compact, yet also disordered configuration is retrieved. In the past, the icosahedral structure has been proposed by studies based on $\mathrm{EAM}^{75,78}$ and Gupta potentials, ${ }^{76}$ but the present configuration is found to be more stable according to DFT $(\Delta E=-0.70 \mathrm{eV})$.

Across the different elements, the structures of the next lowenergy isomers generally resemble those of the putative ground states, i.e., perturbed icosahedra in case of an icosahedral ground state and similarly disordered variations otherwise (see Figure 5). The energy gap between the lowest- and next-

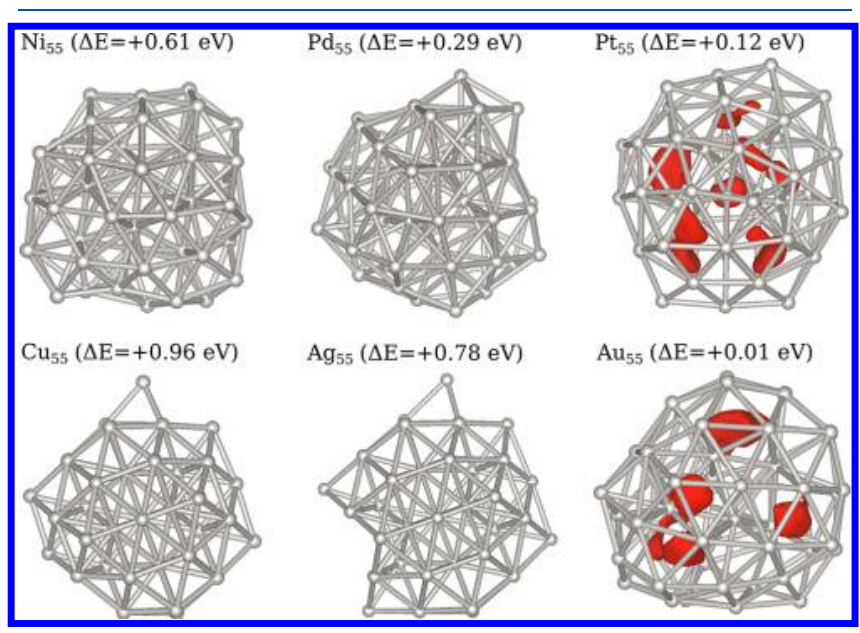

Figure 5. Next-lowest-energy structures obtained among the 55-atom clusters after DFT reoptimization, together with the energy differences relative to the respective ground states. Cavities in the $\mathrm{Pt}_{55}$ and $\mathrm{Au}_{55}$ structures are highlighted by means of electron density isosurfaces (colored in red).

lowest-energy structures is significantly larger in the former case $(0.6-1.0 \mathrm{eV})$ than in the latter $(<0.3 \mathrm{eV}) . \mathrm{Ni}_{55}$ appears to present an exception in the sense that disordered structures are competitive with perturbed icosahedra in terms of the total energy. Given the ferromagnetic properties of nickel, it is appropriate to consider the influence of spin polarization on the energetical ordering. As shown in Table 1, magnetization further increases the relative stability of the Mackay icosahedron, while decreasing that of the disordered isomer.

\section{CONCLUSIONS}

A combined DFTB parametrization and global optimization strategy is presented for the study of metal cluster structures. Starting from DFTB parameters derived from bulk metal properties, the GO algorithm allows to generate cluster
Table 1. Relative Energies (in eV) of the Two Next-LowestEnergy Isomers of $\mathrm{Ni}_{55}$ with Respect to the Mackay Icosahedron, Calculated with and without Spin Polarization Effects

\begin{tabular}{lcc}
\multicolumn{1}{c}{ structure } & nonmagnetic & ferromagnetic \\
disordered structure & 0.61 & 1.77 \\
perturbed icosahedron & 0.89 & 1.23 \\
\hline
\end{tabular}

structures whose DFT energetics provide the basis for a more accurate subsequent DFTB parametrization. As a result, nanometer-sized clusters can be investigated with relative ease.

Application to the 13- and 55-atom clusters of the late transition metals confirms the preference for the icosahedral structure for $\mathrm{Ni}_{13}, \mathrm{Ni}_{55}, \mathrm{Cu}_{55}$, and $\mathrm{Ag}_{55}$ clusters, whereas, e.g., the lowest-energy $\mathrm{M}_{55}$ clusters of $\mathrm{Pd}, \mathrm{Pt}$, and $\mathrm{Au}$ are strongly disordered. While the $\mathrm{Pd}_{55}$ structures remain compact, the previously reported ${ }^{27}$ subsurface cavities in $\mathrm{Au}_{55}$ are here also observed in $\mathrm{Pt}_{55}$.

The present approach hence facilitates the use of more realistic cluster models in, e.g., heterogeneous catalysis research, where high-symmetry structures have for example been frequently used for nanometer-sized Pd and Pt clusters. Promising further applications and developments of the method include the effects of alloying and of deposition on support materials.

\section{ASSOCIATED CONTENT}

\section{Supporting Information}

The Supporting Information is available free of charge on the ACS Publications website at DOI: 10.1021/acs.jpca.9b00927.

Confinement parameters, band structures, repulsive potentials, parity diagrams, and transferability (PDF) Slater-Koster files in DFTB+ format containing the final parameters for each cluster type (ZIP)

Coordinates in $X Y Z$ format of the five lowest-energy structures after DFT reoptimization for each cluster type (ZIP)

\section{AUTHOR INFORMATION}

\section{Corresponding Author}

*(M.V.d.B.) E-mail: maxime.vandenbossche@insp.jussieu.fr. ORCID $\odot$

Maxime Van den Bossche: 0000-0002-0938-0156

Notes

The author declares no competing financial interest.

\section{ACKNOWLEDGMENTS}

The author thanks Vitae Industries for providing computational resources and Prof. Hannes Jónsson, Kusse Sukuta, Prof. Lai-Sheng Wang, and Prof. Andrew Peterson for valuable discussions.

\section{REFERENCES}

(1) Johnston, R. L. Metal Nanoparticles and Nanoalloys. Front. Nanosci. 2012, 3, 1-42.

(2) Fernando, A.; Weerawardene, K. L. D. M.; Karimova, N. V.; Aikens, C. M. Quantum Mechanical Studies of Large Metal, Metal Oxide, and Metal Chalcogenide Nanoparticles and Clusters. Chem. Rev. 2015, 115, 6112-6216.

(3) Choudhary, T. V.; Goodman, D. W. Oxidation Catalysis by Supported Gold Nano-Clusters. Top. Catal. 2002, 21, 25-34. 
(4) Sarwar, M.; Cooper, C.; Briquet, L.; Ukpong, A.; Perry, C.; Jones, G. Atomic-Scale Modelling and its Application to Catalytic Materials Science. Iohnson Matthev Technol. Rev. 2015, 59, 257-283.

(5) Baletto, F.; Ferrando, R. Structural Properties of Nanoclusters: Energetic, Thermodynamic, and Kinetic Effects. Rev. Mod. Phvs. 2005, 77, 371-423.

(6) Heiles, S.; Johnston, R. L. Global Optimization of Clusters Using Electronic Structure Methods. Int. I. Quantum Chem. 2013, 113, 2091-2109.

(7) Trinchero, A.; Klacar, S.; Paz-Borbón, L. O.; Hellman, A.; Grönbeck, H. Oxidation at the Subnanometer Scale. I.Phvs. Chem. C 2015, 119, 10797-10803.

(8) Heard, C. J.; Heiles, S.; Vajda, S.; Johnston, R. L. $\mathrm{Pd}_{\mathrm{n}} \mathrm{Ag}_{(4-\mathrm{n})}$ and $\mathrm{Pd}_{\mathrm{n}} \mathrm{Pt}_{(4-\mathrm{n})}$ Clusters on $\mathrm{MgO}$ (100): a Density Functional Surface Genetic Algorithm Investigation. Nanoscale 2014, 6, 11777-11788.

(9) Hussein, H. A.; Davis, J. B. A.; Johnston, R. L. DFT Global Optimisation of Gas-Phase and MgO-Supported Sub-Nanometre AuPd Clusters. Phvs. Chem. Chem. Phvs. 2016, 18, 26133-26143.

(10) Vilhelmsen, L. B.; Hammer, B. Systematic Study of $\mathrm{Au}_{6}$ to $\mathrm{Au}_{12}$ Gold Clusters on $\mathrm{MgO}(100) \mathrm{F}$ Centers Using Density-Functional Theory. Phvs. Rev. Lett. 2012, 108, 126101.

(11) Johnston, R. L. Evolving Better Nanoparticles: Genetic Algorithms for Optimising Cluster Geometries. Dalton Trans. 2003, 4193-4207.

(12) Lyakhov, A. O.; Oganov, A. R.; Stokes, H. T.; Zhu, Q. New Developments in Evolutionary Structure Prediction Algorithm USPEX. Comput. Phvs. Commun. 2013, 184, 1172-1182.

(13) Meldgaard, S. A.; Kolsbjerg, E. L.; Hammer, B. Machine Learning Enhanced Global Optimization by Clustering Local Environments to Enable Bundled Atomic Energies. I. Chem. Phys. 2018, 149, 134104.

(14) Zhai, H.; Alexandrova, A. N. Ensemble-Average Representation of Pt Clusters in Conditions of Catalysis Accessed through GPU Accelerated Deep Neural Network Fitting Global Optimization. I. Chem. Theory Comput. 2016, 12, 6213-6226.

(15) Kolsbjerg, E. L.; Peterson, A. A.; Hammer, B. Neural-NetworkEnhanced Evolutionary Algorithm Applied to Supported Metal Nanoparticles. Phvs. Rev. B: Condens. Matter Mater. Phvs. 2018, 97, 195424.

(16) Elstner, M.; Seifert, G. Density Functional Tight Binding. Philos. Trans. R. Soc., A 2014, 372, 20120483.

(17) Gaus, M.; Chou, C.-P.; Witek, H.; Elstner, M. Automatized Parametrization of SCC-DFTB Repulsive Potentials: Application to Hydrocarbons. I. Phys. Chem. A 2009, 113, 11866-11881.

(18) Huran, A. W.; Steigemann, C.; Frauenheim, T.; Aradi, B.; Marques, M. A. L. Efficient Automatized Density-Functional TightBinding Parametrizations: Application to Group IV Elements. $\underline{\text { I. Chem. }}$ Theorv Comput. 2018, 14, 2947-2954.

(19) Bobadova-Parvanova, P.; Jackson, K. A.; Srinivas, S.; Horoi, M.; Köhler, C.; Seifert, G. Scanning the Potential Energy Surface of Iron Clusters: A Novel Search Strategy. I. Chem. Phvs. 2002, 116, 35763587.

(20) Köhler, C.; Seifert, G.; Frauenheim, T. Density functional based calculations for $\mathrm{Fe}_{n}(n \leq 32$. Chem. Phvs. 2005, 309, 23-31.

(21) Köhler, C.; Frauenheim, T. Magnetic Moment of Iron Clusters with 109, 110, 111, and 147 Atoms. I. Comput. Theor. Nanosci. 2007, 4, 264-269.

(22) Aktürk, A.; Sebetci, A. BH-DFTB/DFT Calculations for Iron Clusters. AIP Adv. 2016, 6, 055103.

(23) Koskinen, P.; Häkkinen, H.; Seifert, G.; Sanna, S.; Frauenheim, T.; Moseler, M. Density-Functional Based Tight-Binding Study of Small Gold Clusters. New I. Phys. 2006, 8, 9.

(24) Mäkinen, V.; Koskinen, P.; Häkkinen, H. Modeling ThiolateProtected Gold Clusters with Density-Functional Tight-Binding. Eur. Phys. J. D 2013, 67, 38.

(25) Fihey, A.; Hettich, C.; Touzeau, J.; Maurel, F.; Perrier, A.; Köhler, C.; Aradi, B.; Frauenheim, T. SCC-DFTB Parameters for Simulating Hybrid Gold-Thiolates Compounds. I. Comput. Chem. 2015, 36, 2075-2087.
(26) Cuny, J.; Tarrat, N.; Spiegelman, F.; Huguenot, A.; Rapacioli, M. Density-Functional Tight-Binding Approach for Metal Clusters, Nanoparticles, Surfaces and Bulk: Application to Silver and Gold. I. Phvs.: Condens. Matter 2018, 30, 303001.

(27) Tarrat, N.; Rapacioli, M.; Cuny, J.; Morillo, J.; Heully, J.-L.; Spiegelman, F. Global Optimization of Neutral and Charged 20- and 55-Atom Silver and Gold Clusters at the DFTB Level. Comput. Theor. Chem. 2017, 1107, 102-114.

(28) Liu, X.; Wahiduzzaman, M.; Oliveira, A. F.; Heine, T.; Salahub, D. R. Density-Functional-Based Tight-Binding Parameterization of Mo, C, H, O and Si for Studying Hydrogenation Reactions on Molybdenum Carbide. Theor. Chem. Acc. 2016, 135, 168.

(29) Shi, H.; Koskinen, P.; Ramasubramaniam, A. Self-Consistent Charge Density-Functional Tight-Binding Parametrization for Pt-Ru Alloys. I. Phys. Chem. A 2017, 121, 2497-2502.

(30) Seifert, G.; Eschrig, H. LCAO-X $\alpha$ Calculations of Transition Metal Clusters. Phvs. Status Solidi B 1985, 127, 573-585.

(31) Elstner, M.; Porezag, D.; Jungnickel, G.; Elsner, J.; Haugk, M.; Frauenheim, T.; Suhai, S.; Seifert, G. Self-Consistent-Charge DensityFunctional Tight-Binding Method for Simulations of Complex Materials Properties. Phvs. Rev. B: Condens. Matter Mater. Phvs. 1998, 58, 7260-7268.

(32) Slater, J. C.; Koster, G. F. Simplified LCAO Method for the Periodic Potential Problem. Phvs. Rev. 1954, 94, 1498-1524.

(33) Koskinen, P.; Mäkinen, V. Density-Functional Tight-Binding for Beginners. Comput. Mater. Sci. 2009, 47, 237-253.

(34) Foulkes, W. M. C.; Haydock, R. Tight-Binding Models and Density-Functional Theory. Phvs. Rev. B: Condens. Matter Mater. Phvs. 1989, 39, 12520-12536.

(35) Van den Bossche, M.; Grönbeck, H.; Hammer, B. TightBinding Approximation-Enhanced Global Optimization. I. Chem. Theory Comput. 2018, 14, 2797-2807.

(36) Schütt, O.; Messmer, P.; Hutter, J.; VandeVondele, J. GPUAccelerated Sparse Matrix-Matrix Multiplication for Linear Scaling Density Functional Theory. Electronic Structure Calculations on Graphics Processing Units 2016, 173-190.

(37) Hutter, J.; Iannuzzi, M.; Schiffmann, F.; VandeVondele, J. CP2K: Atomistic Simulations of Condensed Matter Systems. WIREs Comput. Mol. Sci. 2014, 4, 15-25.

(38) Borštnik, U.; VandeVondele, J.; Weber, V.; Hutter, J. Sparse Matrix Multiplication: The Distributed Block-Compressed Sparse Row Library. Parallel Comput. 2014, 40, 47-58.

(39) Hess, B. A. Relativistic Electronic-Structure Calculations Employing a Two-Component No-Pair Formalism with ExternalField Projection Operators. Phvs. Rev. A: At. Mol.. Opt. Phvs. 1986, 33, $3742-3748$

(40) Jansen, G.; Hess, B. A. Revision of the Douglas-Kroll Transformation. Phvs. Rev. A: At., Mol., Opt. Phvs. 1989, 39, 60166017.

(41) Douglas, M.; Kroll, N. M. Quantum Electrodynamical Corrections to the Fine Structure of Helium. Ann. Phvs. 1974, 82, $89-155$.

(42) Powell, M. J. D. A Direct Search Optimization Method That Models the Objective and Constraint Functions by Linear Interpolation. Advances in Optimization and Numerical Analvsis 1994, 51-67.

(43) Mortensen, J. J.; Hansen, L. B.; Jacobsen, K. W. Real-Space Grid Implementation of the Projector Augmented Wave Method. Phvs. Rev. B: Condens. Matter Mater. Phvs. 2005, 71, 035109.

(44) Enkovaara, J.; Rostgaard, C.; Mortensen, J. J.; Chen, J.; Dulak, M.; Ferrighi, L.; Gavnholt, J.; Glinsvad, C.; Haikola, V.; Hansen, H. A.; et al. Electronic Structure Calculations with GPAW: A Real-Space Implementation of the Projector Augmented-Wave Method. I. Phvs.: Condens. Matter 2010, 22, 253202.

(45) Perdew, J. P.; Ruzsinszky, A.; Csonka, G. I.; Vydrov, O. A.; Scuseria, G. E.; Constantin, L. A.; Zhou, X.; Burke, K. Restoring the Density-Gradient Expansion for Exchange in Solids and Surfaces. Phys. Rev. Lett. 2008, 100, 136406. 
(46) Johansson, M. P.; Lechtken, A.; Schooss, D.; Kappes, M. M.; Furche, F. 2D-3D Transition of Gold Cluster Anions Resolved. Phys. Rev. A: At., Mol., Opt. Phvs. 2008, 77, 053202.

(47) Fishman, M.; Zhuang, H. L.; Mathew, K.; Dirschka, W.; Hennig, R. G. Accuracy of Exchange-Correlation Functionals and Effect of Solvation on the Surface Energy of Copper. Phys. Rev. B: Condens. Matter Mater. Phvs. 2013, 87, 245402.

(48) Zhuang, H.; Tkalych, A. J.; Carter, E. A. Surface Energy as a Descriptor of Catalytic Activity. L. Phys. Chem. C 2016, 120, 2369823706.

(49) Blöchl, P. E. Projector Augmented-Wave Method. Phys. Rev. B: Condens. Matter Mater. Phvs. 1994, 50, 17953-17979.

(50) Aradi, B.; Hourahine, B.; Frauenheim, T. DFTB+, a Sparse Matrix-Based Implementation of the DFTB Method. I. Phvs. Chem. A 2007, 111, 5678-5684.

(51) Vilhelmsen, L. B.; Hammer, B. A Genetic Algorithm for First Principles Global Structure Optimization of Supported Nano Structures. I. Chem. Phvs. 2014, 141, 044711.

(52) Bahn, S. R.; Jacobsen, K. W. An object-oriented scripting interface to a legacy electronic structure code. Comput. Sci. Eng. 2002, 4, 56-66.

(53) Larsen, A. H.; Mortensen, J. J.; Blomqvist, J.; Castelli, I. E.; Christensen, R.; Dułak, M.; Friis, J.; Groves, M. N.; Hammer, B.; Hargus, C.; et al. Atomic Simulation Environment - a Python Library for Working with Atoms. I. Phvs.: Condens. Matter 2017, 29, 273002.

(54) Head, J. D.; Zerner, M. C. A Broyden-Fletcher-GoldfarbShanno Optimization Procedure for Molecular Geometries. Chem. Phvs. Lett. 1985, 122, 264-270.

(55) Porezag, D.; Frauenheim, T.; Köhler, T.; Seifert, G.; Kaschner, R. Construction of Tight-Binding-Like Potentials on the Basis of Density-Functional Theory: Application to Carbon. Phys. Rev. B: Condens. Matter Mater. Phys. 1995, 51, 12947-12957.

(56) Frauenheim, T.; Seifert, G.; Elsterner, M.; Hajnal, Z.; Jungnickel, G.; Porezag, D.; Suhai, S.; Scholz, R. A Self-Consistent Charge Density-Functional Based Tight-Binding Method for Predictive Materials Simulations in Physics, Chemistry and Biology. Phvs. Status Solidi B 2000, 217, 41-62.

(57) Momma, K.; Izumi, F. VESTA 3 for Three-Dimensional Visualization of Crystal, Volumetric and Morphology Data. I. Appl. Crystallogr. 2011, 44, 1272-1276.

(58) Posada-Amarillas, A.; Garzón, I. L. Vibrational analysis of $\mathrm{Ni}_{55}$ clusters. Phvs. Rev. B: Condens. Matter Mater. Phvs. 1996, 54, 1036210365.

(59) Oviedo, J.; Palmer, R. E. Amorphous Structures of $\mathrm{Cu}, \mathrm{Ag}$, and $\mathrm{Au}$ Nanoclusters from First Principles Calculations. I. Chem. Phys. 2002, 117, 9548-9551.

(60) Köster, A. M.; Calaminici, P.; Orgaz, E.; Roy, D. R.; Reveles, J. U.; Khanna, S. N. On the Ground State of $\mathrm{Pd}_{13}$. I. Am. Chem. Soc. 2011, 133, 12192-12196.

(61) Wang, L.-L.; Johnson, D. D. Density Functional Study of Structural Trends for Late-Transition-Metal 13-Atom Clusters. Phvs. Rev. B: Condens. Matter Mater. Phvs. 2007, 75, 235405.

(62) Ferrighi, L.; Hammer, B.; Madsen, G. K. H. 2D-3D Transition for Cationic and Anionic Gold Clusters: A Kinetic Energy Density Functional Study. I. Am. Chem. Soc. 2009, 131, 10605-10609.

(63) Assadollahzadeh, B.; Schwerdtfeger, P. A Systematic Search for Minimum Structures of Small Gold Clusters $\mathrm{Au}_{\mathrm{n}}(\mathrm{n}=2-20)$ and Their Electronic Properties. L. Chem. Phys. 2009, 131, 064306.

(64) Johansson, M. P.; Warnke, I.; Le, A.; Furche, F. At What Size Do Neutral Gold Clusters Turn Three-Dimensional? I. Phvs. Chem. C 2014, 118, 29370-29377.

(65) Tarrat, N.; Rapacioli, M.; Spiegelman, F. $\mathrm{Au}_{147}$ Nanoparticles: Ordered or Amorphous? I. Chem. Phvs. 2018, 148, 204308.

(66) Häkkinen, H.; Moseler, M.; Kostko, O.; Morgner, N.; Hoffmann, M. A.; von Issendorff, B. Symmetry and Electronic Structure of Noble-Metal Nanoparticles and the Role of Relativity. Phvs. Rev. Lett. 2004, 93, 093401.
(67) Häkkinen, H.; Moseler, M. 55-Atom Clusters of Silver and Gold: Symmetry Breaking by Relativistic Effects. Comput. Mater. Sci. 2006, 35, 332-336.

(68) Liu, S.-R.; Zhai, H.-J.; Wang, L.-S. Evolution of the Electronic Properties of Small $\mathrm{Ni}^{-}{ }_{\mathrm{n}}(\mathrm{n}=1-100)$ Clusters by Photoelectron Spectroscopy. I. Chem. Phys. 2002, 117, 9758-9765.

(69) Michaelian, K.; Rendón, N.; Garzón, I. L. Structure and Energetics of $\mathrm{Ni}, \mathrm{Ag}$, and $\mathrm{Au}$ Nanoclusters. Phys. Rev. B: Condens. Matter Mater. Phvs. 1999, 60, 2000-2010.

(70) Sarkar, U.; Blundell, S. A. Structure and thermodynamics of $\mathrm{Fe}_{55}, \mathrm{Co}_{55}$, and $\mathrm{Ni}_{55}$ clusters supported on a surface. Phvs. Rev. B: Condens. Matter Mater. Phvs. 2009, 79, 125441.

(71) Huang, W.; Ji, M.; Dong, C.-D.; Gu, X.; Wang, L.-M.; Gong, X. G.; Wang, L.-S. Relativistic Effects and the Unique Low-Symmetry Structures of Gold Nanoclusters. ACS Nano 2008, 2, 897-904.

(72) Garzón, I. L.; Posada-Amarillas, A. Structural and vibrational analysis of amorphous $\mathrm{Au}_{55}$ clusters. Phvs. Rev. B: Condens. Matter Mater. Phys. 1996, 54, 11796-11802.

(73) Gorin, D. J.; Toste, F. D. Relativistic Effects in Homogeneous Gold Catalysis. Nature 2007, 446, 395-403.

(74) Sebetci, A.; Güvenç, Z. B. Global Minima for Free Pt $t_{n}$ Clusters $(\mathrm{n}=22-56)$ : A Comparison Between the Searches with a Molecular Dynamics Approach and a Basin-Hopping Algorithm. Eur. Phys.I.D 2004, 30, 71-79.

(75) Sachdev, A.; Masel, R. I.; Adams, J. B. An Embedded Atom Method Study of the Equilibrium Shapes of Small Platinum and Palladium Clusters. Z. Phys. D: At., Mol. Clusters 1993, 26, 310-312.

(76) Massen, C.; Mortimer-Jones, T. V.; Johnston, R. L. Geometries and Segregation Properties of Platinum-Palladium Nanoalloy Clusters. I. Chem. Soc. Dalton Trans. 2002, 0, 4375-4388.

(77) Shi, H.; Auerbach, S. M.; Ramasubramaniam, A. First-Principles Predictions of Structure-Function Relationships of GrapheneSupported Platinum Nanoclusters. L. Phys. Chem. C 2016, 120, 11899-11909.

(78) Karabacak, M.; Özçelik, S.; Güvenç, Z. B. Structures and Energetics of $\mathrm{Pd}_{21}-\mathrm{Pd}_{55}$ Clusters. Surf. Sci. 2003, 532-535, 306-311.

(79) Hotcent is a Python module publicly available at gitlab.com/ $\mathrm{mvdb} /$ hotcent and is based on parts of the Hotbit ${ }^{33}$ program written by Pekka Koskinen.

(80) TANGO is a Python implementation of the TANGO approach and is publicly available at gitlab.com/mvdb/tango 